\title{
Irinotecan-induced Immune Thrombocytopenia
}

\author{
Barry C. Mirtsching, MD, \\ Medical City Dallas Hospital, Dallas, Texas \\ James N. George, MD, \\ Department of Medicine, College of Medicine, University of Oklahoma Health Sciences Center, \\ Oklahoma City, Oklahoma
}

Richard H. Aster, MD, and

Platelet and Neutrophil Immunology Laboratory and Blood Research Institute, Blood Center of Wisconsin and Medical College of Wisconsin, Milwaukee, Wisconsin

\section{Brian R. Curtis, PhD}

Platelet and Neutrophil Immunology Laboratory and Blood Research Institute, Blood Center of Wisconsin and Medical College of Wisconsin, Milwaukee, Wisconsin

Acute immune-mediated thrombocytopenia caused by drugs is well described but commonly misdiagnosed, ${ }^{1}$ because clinicians fail to consider drug exposure as a causative factor. As a consequence, it is not uncommon for patients to experience recurrent episodes of severe thrombocytopenia upon repeated exposure to drug and to receive inappropriate therapy. It is especially likely for a chemotherapeutic agent to be overlooked as a cause of acute immunemediated thrombocytopenia because of the known tendency of this class of drugs to cause thrombocytopenia related as a consequence of marrow suppression. To date, at least 4 chemotherapeutic agents have been documented as triggers for drug-induced immune thrombocytopenia: fludarabine, ${ }^{2}$ dactinomycin, ${ }^{3}$ oxaliplatin ${ }^{4,5}$ and irinotecan. ${ }^{6} \mathrm{We}$ describe here the 2nd reported patient with acute, severe immune-mediated thrombocytopenia caused by irinotecan-dependent platelet-reactive antibodies.

A 53-year-old white woman presented with symptoms of partial colonic obstruction. Colon cancer with multiple liver metastases was diagnosed; no other metastases were detected. Carcinoembryonic antigen (CEA) was $6889 \mu \mathrm{g} / \mathrm{L}$. Treatment with FOLFOX (oxaliplatin, 5fluorouracil and leucovorin) was begun and continued for 11 two-week cycles. Her platelet count before starting FOLFOX was $347,000 / \mu \mathrm{L}$. Bevacizumab was added to the FOLFOX regimen when her obstructive symptoms resolved and the potential risk for emergent surgery had passed. FOLFOX treatment required dose reduction and then discontinuation for modest thrombocytopenia (platelet counts, $84,000-100,000 / \mu \mathrm{L}$ ). On recovery of the platelet count to $143,000 / \mu \mathrm{L}$, transverse colectomy was performed with residual tumor of 2 $\mathrm{cm}$ and 3/14 regional nodes positive for carcinoma. After surgery, treatment continued with bevacizumab-FOLFIRI (irinotecan, 5-fluorouracil and leucovorin) for 6 two-week cycles

Copyright $(\odot$ by the Southern Society for Clinical Investigation.

The other authors have no financial or conflict of interest to disclose. 
(bevacizumab was omitted in the final 2 cycles) when the liver metastases were potentially amenable to resection or local ablation. Platelet counts were maintained between 113,000$150,000 / \mu \mathrm{L}$ during FOLFIRI therapy. CEA at the end of therapy was $9.1 \mu \mathrm{g} / \mathrm{L}$. One year after presentation, the remaining liver lesions were treated with left hepatic segmental hepatectomy and radiofrequency ablation, and a hepatic arterial infusion pump was placed. Hepatic arterial floxuridine therapy was initiated (14 days on, 14 days off) approximately 1 month after surgery and continued for 2 months, when multiple lung metastases were discovered. CEA rose to $14 \mu \mathrm{g} / \mathrm{L}$.

One month later, bevacizumab-FOLFIRI was resumed. The pretreatment platelet count was $150,000 / \mu \mathrm{L}$. Bevacizumab was administered over 90 minutes without incident, followed by irinotecan $\left(180 \mathrm{mg} / \mathrm{m}^{2}\right.$ [340 mg]) over 90 minutes, and then leucovorin over 120 minutes. At the completion of the leucovorin administration, the patient developed a sinus tachycardia of 180-200/min, with stable blood pressure. 5-Fluorouracil administration was withheld. The tachycardia spontaneously terminated within 15 minutes. At that time, her platelet count was $2000 / \mu \mathrm{L}$, verified by repeat analysis (Figure 1A). The patient's examination was normal; she had no signs or symptoms of bleeding. Port and infusion pump contents were immediately emptied and filled with saline. Other laboratory data included: white blood cells $12,900 / \mu \mathrm{L}$ (96\% granulocytes, $2 \%$ lymphocytes and $2 \%$ monocytes), hemoglobin $13.8 \mathrm{~g} / \mathrm{dL}$ (no schistocytes present in blood smear), D-dimer $1.07 \mathrm{ng} / \mathrm{mL}$ (normal, $0.17-0.59 \mathrm{ng} / \mathrm{mL}$ ), INR 0.9, PTT 25.2 seconds, fibrinogen $321 \mathrm{mg} / \mathrm{dL}$, creatinine $0.7 \mathrm{mg} / \mathrm{dL}$, AST $22 \mathrm{IU} / \mathrm{L}$, ALT 29 IU/L and LDH 193 IU/L.

Laboratory tests for heparin-dependent antibodies by both PF4 enzyme-linked immunosorbent assay and serotonin release assay were negative. Testing for drug-dependent platelet antibodies using a previously described flow cytometry assay ${ }^{7}$ was negative for bevacizumab, 5-flurouracil, leucovorin and oxaliplatin (results not shown). However, strong IgG platelet-reactive antibodies were detected in the patient's acute serum only in the presence of irinotecan (Figure 1B), and not with normal control serum and drug (Figure 1C). The patient was observed without specific therapy and her platelet count increased over the ensuing 2 weeks to normal levels (Figure 1A). There was no recurrence of tachycardia. Treatment with bevacizumab, 5-fluorouracil and leucovorin, including the premedications with palonosetron, dexamethasone and atropine, was resumed without incident.

Irinotecan, a synthetic analog of camptothecin, is a topoisomerase I inhibitor often given in combination with 5-fluorouracil, leucovorin and irinotecan (FOLFIRI) for the treatment of metastatic colorectal cancer. Irinotecan is metabolized to its active metabolite, $\mathrm{SN}-38$, which is $1000 \times$ more active than the unmodified drug. Common side effects include diarrhea and nausea/vomiting. Neutropenia resulting from myelosuppression is the dominant hematologic side effect and thrombocytopenia, when it occurs, is usually mild.

In the present case, irinotecan was implicated as the cause of acute immune-mediated thrombocytopenia by both clinical criteria and by identification of drug-dependent platelet antibodies. The patient had apparently become sensitized to irinotecan during the initial 6 cycles of FOLFIRI. She had no platelet counts less than $100,000 / \mu \mathrm{L}$ and no systemic symptoms during her previous exposures to the drug. Her only symptom during the acute 
irinotecan-induced thrombocytopenia was transient sinus tachycardia. A search of MEDLINE and PubMed on June 3, 2013, identified only 1 previous report of irinotecaninduced thrombocytopenia. ${ }^{6}$ In this patient, the irinotecan etiology was confirmed by recurrence of acute severe thrombocytopenia after a repeat irinotecan infusion and by detection of irinotecan-dependent platelet antibodies using a platelet indirect immunofluorescence test. ${ }^{6} \mathrm{We}$ have previously identified irinotecan-dependent antibodies in 2 patients in whom irinotecan-induced thrombocytopenia was suspected (BR Curtis and RH Aster; unpublished observations). Each patient experienced severe thrombocytopenia (5000 and 20,000 platelets per microliter, respectively) within a few hours of being treated for metastatic carcinoma with combination chemotherapy that included irinotecan, and both patients regained a normal platelet count within 1 week without specific intervention. However, drug-dependent reactions of these 2 antibodies with normal platelets were much weaker than those obtained with antibody from the patient described here. The active form of irinotecan is conjugated to glucuronide, and it is excreted mainly in that form. Glucuronide conjugates of many drugs are capable of inducing drug-induced immune thrombocytopenia, and the responsible antibodies react best when tested with the sensitizing metabolite. It is therefore possible that the weak drug-dependent antibody (DDAb) reactions observed with the 2 patients identified previously were due to metabolite-specific antibodies, but testing for metabolite-specific antibodies was not performed. Metabolitedependent platelet antibodies were not detected in serum from the single case of irinotecaninduced thrombocytopenia reported previously when serum was tested against platelets in the presence of the SN-38 metabolite. ${ }^{6}$

Because 4 cases of acute, severe thrombocytopenia caused by irinotecan have now been identified, clinicians should consider this possible diagnosis in any patient who experiences an acute and isolated drop in the platelet levels after irinotecan administration. As standard treatment in cases of thrombocytopenia caused by drugs, transfusion would not be recommended in the absence of any bleeding; observation alone is recommended because spontaneous recovery of the platelet counts is expected after discontinuation of the drug. Steroid therapy should only be considered in circumstances of bleeding, or persistence of severe thrombocytopenia (e.g. platelelets $\leq 10,000 / \mu \mathrm{L}$ ) after some period of observation.

\section{Acknowledgments}

RHA currently receives NIH Grant HL-13629 from NHLBI. BRC was a past consultant for Immucor, Inc, and served in past on PNH Advisory Board of Alexion Pharmaceutical.

\section{References}

1. Aster RH, Bougie DW. Drug-induced immune thrombocytopenia. N Engl J Med. 2007; 357:580-7. [PubMed: 17687133]

2. Fernandez MJ, Llopis I, Pastor E, et al. Immune thrombocytopenia induced by fludarabine successfully treated with rituximab. Haematologica. 2003; 88:ELT02. [PubMed: 12604433]

3. Khatua S, Nair CN, Ghosh K. Immune-mediated thrombocytopenia following dactinomycin therapy in a child with alveolar rhabdomyosarcoma: the unresolved issues. J Pediatr Hematol Oncol. 2004; 26:777-9. [PubMed: 15543019]

4. Curtis BR, Kaliszewski J, Marques MB, et al. Immune-mediated thrombocytopenia resulting from sensitivity to oxaliplatin. Am J Hematol. 2006; 81:193-8. [PubMed: 16493620] 
5. Jardim DL, Rodrigues CA, Novis YA, et al. Oxaliplatin-related thrombocytopenia. Ann Oncol. 2012; 23:1937-42. [PubMed: 22534771]

6. Bozec L, Bierling P, Fromont P, et al. Irinotecan-induced immune thrombocytopenia. Ann Oncol. 1998; 9:453-5. [PubMed: 9636839]

7. Curtis BR, McFarland JG, Wu GG, et al. Antibodies in sulfonamide-induced immune thrombocytopenia recognize calcium-dependent epitopes on the glycoprotein IIb/IIIa complex. Blood. 1994; 84:176-83. [PubMed: 7517207] 


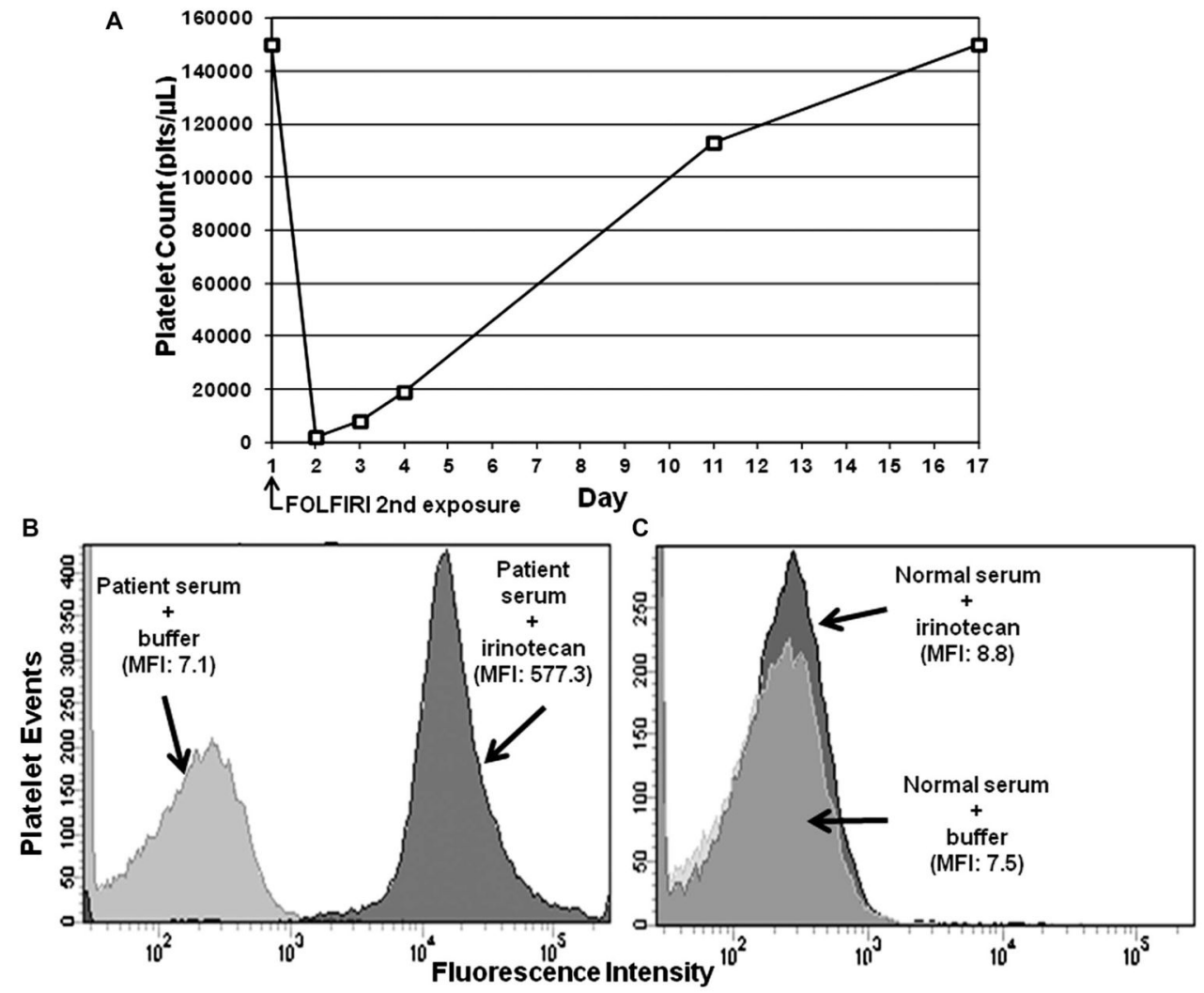

FIGURE 1.

(A) Graph of the patient's platelet levels just before and after bevacizumab-FOLFIRI administration was resumed. Testing of patient's serum for drug-dependent platelet antibodies by flow cytometry assay showed strong $\mathrm{IgG}$ antibody reactivity $(\mathrm{MFI}=577.3)$ in the patient's acute serum only in the presence of irinotecan (B) (dark histogram), but not with normal control serum and drug (C) (dark histogram). MFI = median fluorescence intensity. 